\title{
Conditions associated with worse acceptance of a simplified accelerated recovery after surgery protocol in laparoscopic colorectal surgery
}

Fábio Lopes de Queiroz ${ }^{1 *}$ (D), Antonio Lacerda-Filho ${ }^{2}$, Adriana Cherem Alves ${ }^{3,4}$, Fábio Henrique de Oliveira ${ }^{5}$, Paulo Rocha França Neto ${ }^{6}$ and Rodrigo de Almeida Paiva ${ }^{6}$

\begin{abstract}
Background: Enhanced Recovery Surgical Programs were initially applied to colorectal procedures and used as multimodal approach to relieve the response to surgical stress. An important factor that negatively impacts the success of these programs is the poor tolerance of these patients to certain items in the adopted protocol, especially with regard to post-operative measures. The identification of these factors may help to increase the success rate of such programs, ensuring that benefits reach a greater number of patients and that resources are better allocated. Thus, the aims of this study were to assess the results of the implementation of a Simplified Accelerated Recovery Protocol (SARP) and to identify possible factors associated with failure to implement postoperative protocol measures in patients submitted to laparoscopic colorectal surgery.
\end{abstract}

Methods: 161 patients were randomly divided into two groups. The SARP group $(n=84)$ was submitted to the accelerated recovery program and the CC group $(n=77)$, to conventional postoperative care. The SARP group was further divided into two subgroups: patients who tolerated the protocol $(n=51)$ and those who did not $(n=33)$, in order to analyze factors contributing to protocol nontolerance.

Results: The groups had similar sociodemographic and clinical characteristics. The SARP group had a shorter hospital stay, better elimination of flatus, was able to walk and to tolerate a diet sooner $(p<0.0001)$. Complications rates and readmissions to emergency room were similar between groups. Multivariate analysis revealed that prolonged operating time, stoma creation and rates of surgical complications were independently associated with poor adherence to $\operatorname{SARP}(p<0.0001)$.

Conclusions: The use of our SARP resulted in improved recovery from laparoscopic colorectal surgery and proved to be safe for patients. Extensive surgeries, occurrence of complications, and the need for ostomy were variables associated with poor program adhesion.

Trial registration Trial Registry: RBR2b4fyr—Date of registration: 03 October 2017.

Keywords: Colectomy, Accelerated postoperative recovery, Perioperative care, Nutrition

*Correspondence: fabiolopesq@hotmail.com

${ }^{1}$ Colorectal Surgery Department, Hospital Felicio Rocho, Rua Felipe Dos Santos, 760, 501-3, Belo Horizonte, Minas Gerais CEP 30180160, Brazil

Full list of author information is available at the end of the article

\section{Background}

Enhanced Recovery After Surgery ${ }^{\mathrm{TM}}$ (ERAS) programs aim to replace traditional perioperative practices, which have long been adopted and used, with interventions that 
have proven to be beneficial, based on solid scientific evidence. ERAS programs were initially applied to colorectal surgery and used a multimodal care approach to relieve the response to surgical stress, accelerating postoperative recovery and reducing costs for both public and private health systems $[1,2]$. However, in spite of their scientifically-proven advantages and of the widespread adoption of minimally-invasive techniques, one their main features, ERAS ${ }^{\mathrm{TM}}$ protocols are used in less than one-third of surgical procedures in the United States and the United Kingdom [2-5]. This is mainly due to operational difficulties, initial costs for implementation and also due to the complexity and a number of interventions advocated by most of the described protocols, which hampers patients acceptance making it difficult to be adopted by many institutions [6,7].

The adoption of simplified protocols, with a smaller number of interventions, could favor the acceptance of such measures not only by patients, but also by a greater number of institutions, mainly in developing countries, where resources are scarce. However, even with the use of less complex protocols a significant percentage of patients did not tolerate the implemented measures. This is true, especially regarding to post-operative measures, which have a non-acceptance rate of up to $40 \%$ [5-8], reducing substantially the expected benefits of adopting the same. The identification of factors related to non-tolerance may help to increase the success rate of such programs, ensuring that benefits reach a greater number of patients and that resources are better allocated. Thus, the aims of this study were to assess the results of the implementation of a Simplified Accelerated Recovery Protocol
(SARP) and to identify possible factors associated with failure to implement postoperative protocol measures in patients submitted to laparoscopic colorectal surgery.

\section{Methods}

This was a prospective randomized study comparing two groups of patients. One was submitted to simplified accelerated recovery protocol (SARP), focusing on postoperative measures (Table 1), while the other group underwent conventional postoperative care (CC) (Table 2). Patients over 18 years of age were included, as per classifications I and II of the American Society of Anesthesiologists (ASA). These patients underwent elective laparoscopic colorectal surgery with anastomosis, with or without protective ostomy. The study excluded patients with end-stage colorectal cancer, mental or psychiatric disorders, and those with contraindications to laparoscopic surgery. Also excluded were pregnant patients and those who had previously undergone esophageal, gastroduodenal, or pancreatic surgery, as well as those who refused to participate in the study. All participants signed a statement of Informed Consent.

The patients underwent surgery at the Colorectal Surgery Department of Felício Rocho Hospital, a tertiary referral hospital in Belo Horizonte, Brazil, over a two-year period. Initially, 250 patients were considered eligible; 71 patients were excluded for not meeting the inclusion criteria, while three were excluded for refusing to participate in the study. Of the 176 participating patients who underwent colorectal resections by videoassisted laparoscopy, 13 (7.4\%) and $2(1.1 \%)$ patients were excluded due to conversion and data collection failures,

Table 1 Simplified accelerated postoperative recovery program (SARP)

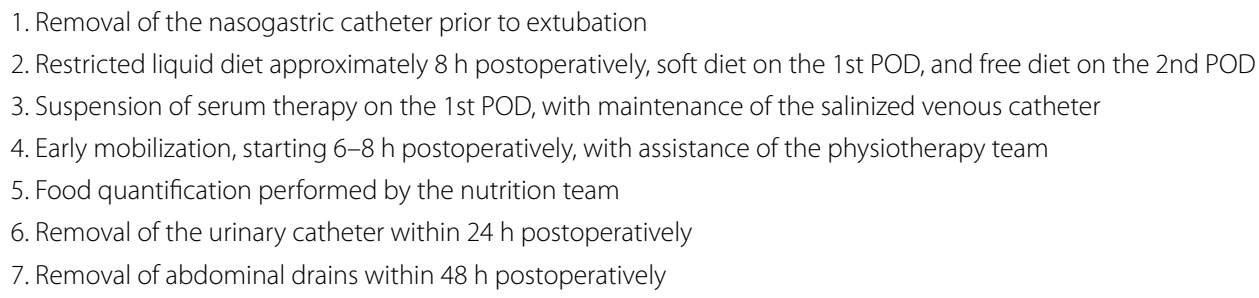

Table 2 Conventional postoperative care (CC)

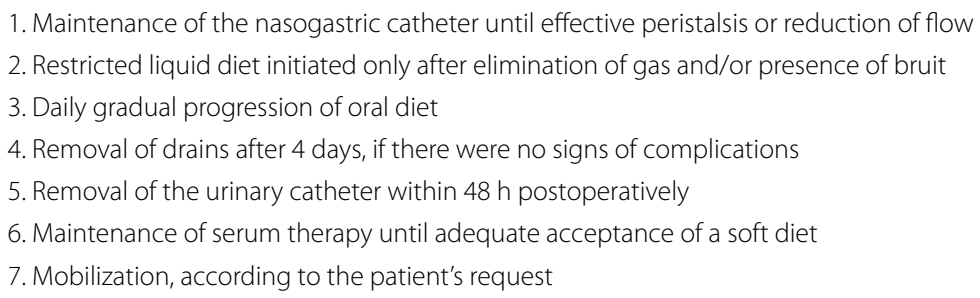


respectively. The remaining 161 patients were included in the data analysis. The distribution of patients and of analyzed groups is presented in the CONSORT diagram [9] (Fig. 1).
Colon preparation was performed with oral sodium phosphate. A regular diet was maintained until eight hours before surgery and patients were encouraged to ingest clear liquids up to three hours before surgery. All

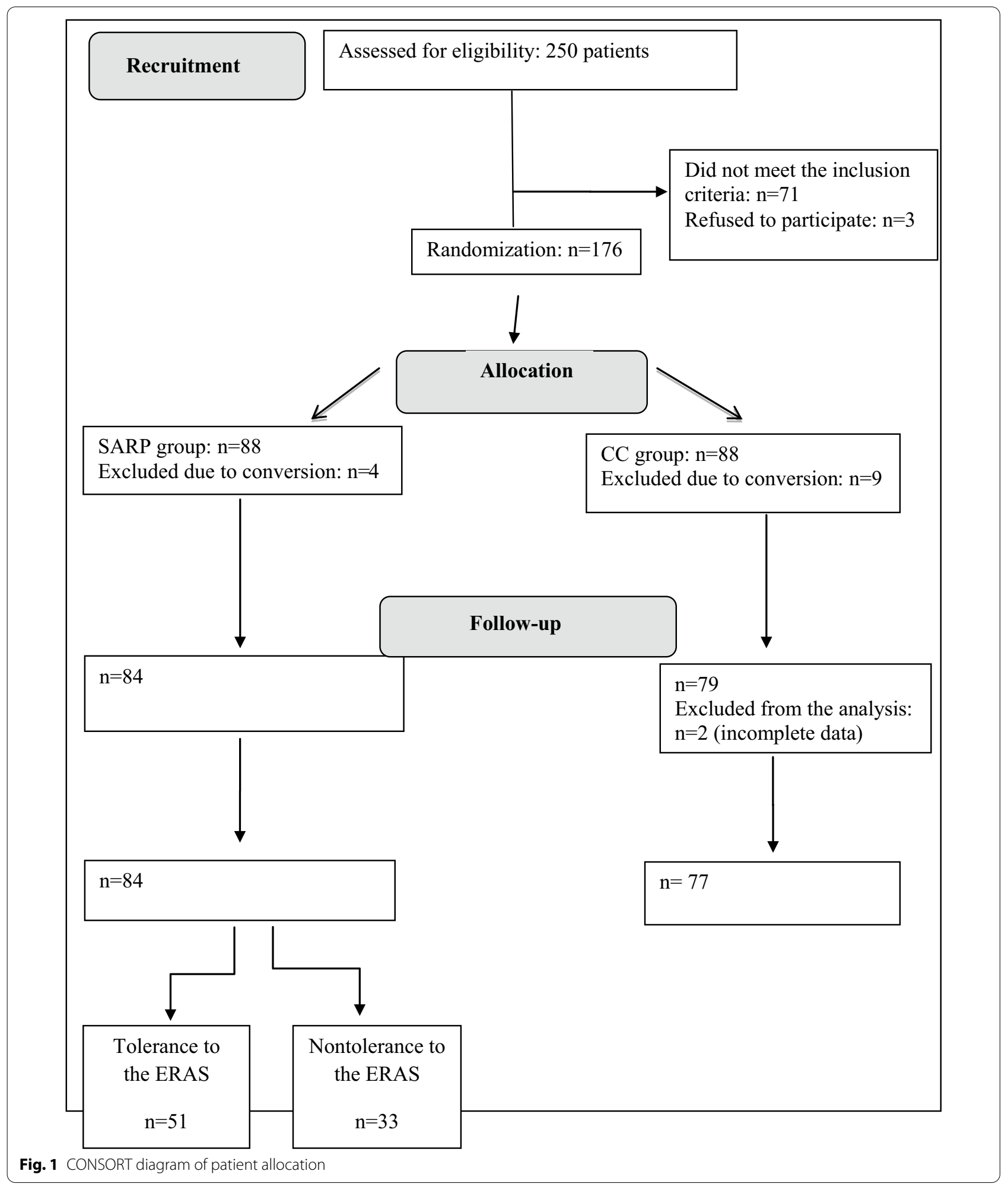


patients received standardized anesthetic care and antimicrobial prophylaxis was administered in a single dose of ceftriaxone $2 \mathrm{~g}$ and metronidazole $1.5 \mathrm{~g}$, thirty minutes before skin incision. Urinary and nasogastric catheters were placed at the start of surgery to monitor urine output and to achieve gastric decompression, respectively.

All procedures were started using video-assisted laparoscopy, with two $10 \mathrm{~mm}$ portals and three $5 \mathrm{~mm}$ portals. Creation of larger than necessary longitudinal or transverse incisions, to allow for the removal of surgical specimens $(>8 \mathrm{~cm})$ was considered to be a conversion. A diverting loop ileostomy was created for all patients undergoing low anterior resection.

At the end of the surgery, the patients underwent simple randomization by drawing numbers 1 (SARP) or 2 (CC) marked on cards in a closed box. Groups were compared to assess their similarities and then they were compared with each other to prove the benefits of the simplified protocol of accelerated recovery. In this case, hospital discharge until the third postoperative day (POD) was considered as a criterion for tolerance to SARP [10-12]. Patients who were not discharged by the third POD, according to these criteria, were considered intolerant to the program. The established criteria for discharge were stable vital signs, an alert and oriented mental status, absence of symptoms and of suspected complications, unassisted walking, tolerance to a soft or solid diet, elimination of flatus, spontaneous diuresis, good pain control with oral analgesics, self-sufficiency in basic daily activities, and the patient's expressed desire to go home.

In order to assess possible factors working against the adoption of the protocol in the SARP group, the "tolerant" subgroup (patients discharged by the third POD) and the "non-tolerant" subgroup (those discharged after the third POD) were compared to each other. The variables potentially involved in tolerance or non-tolerance to program items were presence of complications, need for ostomy, operative time, body mass index (BMI), age, sex, ASA classification 1 or 2, presence of comorbidities, malignant or benign disease, and type of surgery. Regarding the type of surgery, two groups were formed: patients who underwent surgery involving only the colon (colectomies) and those who underwent surgeries that involved rectal resection that is total or partial mesorectal excison.

Upon discharge, oral and antispasmodic analgesics (hyoscine and dipyrone or paracetamol) were prescribed to the patients, and the use of enoxaparin at $40 \mathrm{mg} / \mathrm{day}$ for four weeks was recommended. Patients were guided on how to contact the team in case of intercurrent events, such as abdominal pain or distension, fever, an interruption in the elimination of flatus and feces, among others. All patients were instructed to return for postoperative consultation seven to ten days after discharge, or as needed.

Postoperative complications were considered to be those that developed by the thirtieth POD, categorized according to the Dindo-Clavien classification [13].

This study was approved by the Research Ethics Committee of our institution and by the Research Ethics Committee of the Federal University of Minas Gerais. It was registered on the Brazilian Platform of Research under CAAE number 43719015.4.0000.5149. It is enrolled in the Brazilian Clinical Trials Registry, linked to the International Clinical Trials Registry of the World Health Organization under number RBR-2b4fyr.

\section{Statistical analysis}

The sample size was 161 determined using Epi Info software version 7.2 , with power equal to 0.80 , a margin of error of $5,02 \%$, an expected tolerance percentage of 0.12 , and a confidence level of $95 \%$. The statistical analysis of the variables was performed using the SPSS program (IBM-version 20.0, 2011). To compare the quantitative measures, the Mann-Whitney $U$ test was used for non-normal distributions, and Student's t-test for normal distributions, with verification by the Shapiro-Wilk test. Pearson's asymptotic and exact chi-square tests were used to compare category variables. Multivariate logistic regression was used to analyze association. Variables with an association at the 0.20 level were considered candidates for the multivariate model. They were jointly analyzed until only those with significance at the 0.05 level remained. The quality of the adjustment was analyzed using the Hosmer-Lemeshow test. A $p$-value $<0.05$ was considered statistically significant.

\section{Results}

Of the 161 patients included in the final analysis, 84 (52.2\%) were allocated to the SARP group and 77 (47.8\%), to the CC group. Ninety-six patients were female (59.6\%). Age ranged from 25 to 95 years, with an average of $57.4 \pm 12.6$ years. BMI ranged from 18.0 to $51.0 \mathrm{~kg} / \mathrm{m}^{2}$, with a median of $25.5 \mathrm{~kg} / \mathrm{m}^{2}$.

The two groups were similar with respect to demographic characteristics, as well as to the type and to the duration of the surgery performed, as shown in Table 3.

The median time of surgical procedures was $240.0 \mathrm{~min}$ and did not differ between groups $(p=0.274)$. The most common surgical indication was colorectal cancer, with 115 cases $(71.4 \%)$. Thirty-seven patients $(23 \%)$ required ostomy.

A total of 39 patients developed complications, with an overall rate of $24.2 \%$. The SARP group presented a rate of $25 \%(21 / 84)$, while the CC group presented a rate of $23.4 \%(18 / 77)(p=0.810)$. One patient in the SARP 
Table 3 Demographic, clinical, and surgical characteristics of patients in the SARP and CC groups $(n=161)$

\begin{tabular}{|c|c|c|c|}
\hline Variables & SARP $(n=84)$ & $C C(n=77)$ & $p$-value \\
\hline \multicolumn{4}{|l|}{ Age } \\
\hline Mean \pm SD & $55.01 \pm 12.60$ & $58.39 \pm 13.86$ & \multirow[t]{2}{*}{$0.074^{\mathrm{a}}$} \\
\hline Median (Q1; Q3) & $55.5(48.0 ; 61.8)$ & $58.0(51.0 ; 70.0)$ & \\
\hline \multicolumn{4}{|l|}{$\operatorname{Sex}, n(\%)$} \\
\hline Female & $51(53.1)$ & $45(46.9)$ & \multirow[t]{2}{*}{$0.769^{b}$} \\
\hline Male & $33(50.8)$ & $32(49.2)$ & \\
\hline \multicolumn{4}{|l|}{$B M l$} \\
\hline Mean \pm SD & $25.41 \pm 5.14$ & $25.89 \pm 4.91$ & \multirow[t]{2}{*}{$0.890^{\mathrm{a}}$} \\
\hline Median (Q1; Q3) & $25.47(22.54 ; 28.41)$ & $25.24(22.60 ; 27.97)$ & \\
\hline \multicolumn{4}{|l|}{$A S A, n(\%)$} \\
\hline 1 & $41(51.9)$ & $38(48.1)$ & \multirow[t]{2}{*}{$0.945^{b}$} \\
\hline 2 & $43(52.4)$ & $39(47.6)$ & \\
\hline \multicolumn{4}{|l|}{ Disease, $n(\%)$} \\
\hline Malignant & $57(49.6)$ & $58(50.4)$ & \multirow[t]{2}{*}{$0.295^{b}$} \\
\hline Benign & $27(58.7)$ & $19(41.3)$ & \\
\hline \multicolumn{4}{|l|}{ Type of surgery, n (\%) } \\
\hline Colectomy & $70(83.33)$ & $59(76.62)$ & \multirow[t]{4}{*}{$0.084^{b}$} \\
\hline Proctectomy & $14(16.67)$ & $18(23.28)$ & \\
\hline Duration of the surgery, min & $234.25 \pm 67.11$ & $249.81 \pm 77.34$ & \\
\hline Mean $\pm S D$ & $240.00(180.00 ; 270.00)$ & $240.00(195.00 ; 300.00)$ & \\
\hline Median (Q1; Q3) & & & $0.274^{\mathrm{a}}$ \\
\hline \multicolumn{4}{|c|}{ Concomitant resection of other organs, $n$ (\%) } \\
\hline Yes & $25(50.0)$ & $25(50.0)$ & \multirow[t]{2}{*}{$0.711^{b}$} \\
\hline No & $59(53.2)$ & $52(46.8)$ & \\
\hline \multicolumn{4}{|l|}{ Need for ostomy, n (\%) } \\
\hline Ileostomy & $14(37.8)$ & $23(62.2)$ & \multirow[t]{2}{*}{$0.061^{c}$} \\
\hline No & $69(56.1)$ & $54(43.9)$ & \\
\hline
\end{tabular}

SARP simplified accelerated postoperative recovery program, CC conventional postoperative care, BMI body mass index, SD standard deviation, Q1; Q3 interquartile range

${ }^{a}$ Mann Whitney U test; ${ }^{b}$ asymptotic chi-square test; ${ }^{c}$ exact chi-square test

group died on the sixth POD due to an anastomotic fistula, resulting in an overall mortality rate of $0.62 \%$. Four patients, with three belonging to the $\mathrm{CC}$ group, returned to the emergency room after discharge and were readmitted to the hospital. One patient presented with an anastomotic fistula, one with dehydration, and two with nausea and vomiting caused by an adynamic ileus. The median length of hospital stay was 3.0 days in the SARP group and 5.0 days in the CC group $(p<0.0001)$. In addition to a shorter hospitalization period, patients in the SARP group started walking, expelling gas, and tolerating a diet sooner. On the other hand, patients in this group relied more heavily on analgesics (Table 4 ).

In the SARP group, 51 of 84 patients were discharged by the third POD, resulting in a success rate of $60 \%$ for the implementation of SARP, according to the criteria adopted in the present study (Table 4).

When comparing the two subgroups in the SARP group, (i.e., patients who tolerated and those who did not tolerate the protocol), univariate analysis demonstrated that the development of complications, the need for ostomy, a longer operative time, and the performance of rectal resection were all factors associated with nontolerance to SARP (Table 5).

In multivariate analysis, the development of complications, a longer operative time, and the need for ostomy were identified as predictors of non-tolerance to SARP items (Table 6).

\section{Discussion}

The simplified accelerated recovery protocol used in this study showed benefits compared to a conventional care protocol, with a median length of hospital stay of three days in the SARP group, significantly lower, than in the conventional group. Furthermore, patients passed gas and tolerated the prescribed diet sooner.

The results obtained with regard to the conversion rate, morbidity, and mortality are all comparable to those 
Table 4 Comparative analysis of postoperative results among patients in the SARP and CC groups $(n=161)$

\begin{tabular}{|c|c|c|c|}
\hline Variables & $\begin{array}{l}\text { SARP }(n=84) \\
n \%\end{array}$ & $\begin{array}{l}C C(n=77) \\
n \%\end{array}$ & $p$-value \\
\hline \multicolumn{4}{|l|}{ Hospitalization time } \\
\hline Median (Q1; Q3) & $3.00(2.0 ; 4.00)$ & $5.00(5.0 ; 7.00)$ & $<0.0001^{\mathrm{a}}$ \\
\hline \multicolumn{4}{|l|}{ Hospital discharge } \\
\hline$\leq 3$ days & $51(60 \%)$ & $0(0.0)$ & $<0.0001^{b}$ \\
\hline$>3$ days & $33(40 \%)$ & $77(100 \%)$ & \\
\hline \multicolumn{4}{|l|}{ Nausea } \\
\hline Yes & $30(44.8)$ & $37(55.2)$ & $0.113^{b}$ \\
\hline No & $54(57.4)$ & $40(42.6)$ & \\
\hline \multicolumn{4}{|l|}{ Vomiting } \\
\hline Yes & $19(46.3)$ & $22(53.7)$ & $0.387^{b}$ \\
\hline No & $65(54.2)$ & $55(45.8)$ & \\
\hline \multicolumn{4}{|l|}{ Walking (days) } \\
\hline 1st POD & $64(65.3)$ & $34(34.7)$ & $<0.0001^{b}$ \\
\hline After 1st POD & $20(31.7)$ & $43(68.3)$ & \\
\hline \multicolumn{4}{|l|}{ Elimination of gas (days) } \\
\hline 1st POD & $49(65.3)$ & $26(34.7)$ & $0.002^{b}$ \\
\hline After 1st POD & $35(40.7)$ & $51(59.3)$ & \\
\hline \multicolumn{4}{|l|}{ Bowel movements } \\
\hline Up to 3rd POD & $56(52.8)$ & $50(47.2)$ & $0.817^{b}$ \\
\hline After 3rd POD & $28(50.9)$ & $27(49.1)$ & \\
\hline \multicolumn{4}{|c|}{ Tolerance to free diet (days) } \\
\hline Up to 3rd POD & $72(97.3)$ & $2(2.7)$ & $<0.0001^{b}$ \\
\hline After 3rd POD & $12(13.8)$ & $75(86.2)$ & \\
\hline \multicolumn{4}{|c|}{ Use of analgesics, anti-inflammatory drugs, and opioids } \\
\hline Yes & $82(54.7)$ & $68(45.3)$ & $0.019^{b}$ \\
\hline No & $2(18.2)$ & $9(81.8)$ & \\
\hline \multicolumn{4}{|c|}{ Return to the hospital and rehospitalization after discharge } \\
\hline Yes & $1(25.0)$ & $3(75.0)$ & $0.350^{c}$ \\
\hline No & $83(52.9)$ & $74(47.1)$ & \\
\hline \multicolumn{4}{|c|}{ Postoperative complications (Clavien-Dindo) } \\
\hline No complications & $63(51.6)$ & $59(48.37)$ & 0.810 \\
\hline Complication, $C D \geq 1$ & $21(53.8)$ & $18(46.2)$ & \\
\hline
\end{tabular}

Bold values indicate better results in patients submitted to the SARP protocol $S A R P$ simplified accelerated recovery program, $C$ conventional postoperative care, $C D$ Clavien-Dindo, $P O D$ postoperative day

${ }^{\mathrm{a}}$ Mann-Whitney $\mathrm{U}$ test; ${ }^{\mathrm{b}}$ asymptotic chi-square test; ${ }^{\mathrm{C}}$ exact chi-square test

of other studies $[14,15]$.Complication and readmission rates, as well as returns to the emergency room, were not different between groups. Therefore, we conclude that the implementation of an accelerated recovery protocol, even if simplified (SARP), was beneficial and safe for patients, as it shortened hospital stays without increasing the risk of complications or rehospitalizations $[10,16]$.

In addition, the present study was able to demonstrate a $60 \%$ acceptance rate for SARP, which matches the rates described in the literature, despite the wide variation observed [7, 8, 14, 17]. Nygren et al. reported the importance of maximizing adherence to ERAS ${ }^{\mathrm{TM}}$ protocols, but this adherence is often hampered by their complexity [7, 14]. Some pilot studies describe more than $90 \%$ acceptance [8], while others observe very low rates of adherence to accelerated recovery programs [17]. The present study, as is the case with a few others described in the literature $[18,19]$, aims to simplify the protocol, adapting it to the actual circumstances of our institution. In this way, a simplified protocol was adopted (Table 1) and, even though it counted only seven items, the success rate remained at $60 \%$. This result demonstrates that four out of ten patients did not benefit from SARP, making it clear that it is still possible to improve the cost-benefit ratio by increasing the acceptance rate of these protocols. The identification of these patients, as well as the reasons for non-tolerance, certainly makes it possible to achieve even better results in the future.

Development of postoperative complications, need for ostomy, a prolonged operative time, and surgeries with rectal resection, were associated with non-tolerance to the program in the univariate analysis. In the multivariate analysis, only development of complications, the need for ostomy, and a prolonged operative time remained as independent factors for a poor adherence to SARP.

Of the patients who had complications, $81 \%$ did not tolerate SARP $(p<0.0001)$. The possible reasons associated with non-tolerance to the program are immobilization over an extended period, greater difficulty in accepting a diet due to a longer ileum recovery, and uncertainty of being discharged after the complication.

Patients who required ostomy had a significantly higher SARP failure rate. Only $14.3 \%$ of patients with ostomy tolerated SARP $(p<0.0001)$, which matches the results from other studies that analyzed this finding [20, 21]. According to Delaney et al [22], the need for ostomy is an independent risk factor for prolonging hospital stay after colorectal surgery. Some studies have shown that setting up an educational program prior to discharge, with guidelines on how to care for the stoma, can shorten the hospital stay. These educational programs are even more effective if performed in the preoperative period, particularly with the involvement of a specialized physician or a stomatherapist [23-25]. Therefore, educational programs are an excellent investment of resources, in order to optimize the acceptance of accelerated postoperative recovery protocols.

Prolonged operative time was another factor that influenced the acceptance of SARP. The mean surgical time in the group that tolerated SARP was significantly lower than the mean in the group that did not. Adoption of measures that reduce surgery length, such as the strict standardization of the surgical technique and the 
Table 5 Results of the univariate analysis of the possible variables that influenced the tolerance or nontolerance to the accelerated recovery program in the subgroups of the SARP group $(n=84)$

\begin{tabular}{|c|c|c|c|}
\hline Variables & $\begin{array}{l}\text { Tolerance to "SARP" } \\
(\mathrm{n}=51)\end{array}$ & $\begin{array}{l}\text { Nontolerance to "SARP" } \\
(\mathrm{n}=33)\end{array}$ & $p$-value \\
\hline \multicolumn{4}{|c|}{ Postoperative complications (Clavien-Dindo), $n$ (\%) } \\
\hline Yes, $C D \geq 1$ & $4(19.0)$ & $17(81)$ & $<0.0001^{b}$ \\
\hline No & $47(74.6)$ & $16(25.4)$ & \\
\hline \multicolumn{4}{|c|}{ Need to perform ostomy, $n$ (\%) } \\
\hline Yes & $2(14.3)$ & $12(85.7)$ & $<0.0001^{b}$ \\
\hline No & $49(70)$ & $21(30.0)$ & \\
\hline Duration of surgery, min & & & $0.004^{d}$ \\
\hline Mean $\pm S D$ & $217.87 \pm 56.86$ & $262.00 \pm 75.89$ & \\
\hline Median (Q1; Q3) & $252.50(210.00 ; 305.00)$ & $210.00(180.00 ; 240.00)$ & \\
\hline \multicolumn{4}{|l|}{ Type of surgery, n (\%) } \\
\hline Colectomy & $49(70.0)$ & $21(30.0)$ & \\
\hline Proctectomy & $2(14.3)$ & $12(85.7)$ & $<0.0001^{\mathrm{b}}$ \\
\hline \multicolumn{4}{|l|}{$B M I, n(\%)$} \\
\hline$<25$ & $14(50)$ & $14(50)$ & $0.069^{b}$ \\
\hline$\geq 25$ & $30(71.4)$ & $12(28.6)$ & \\
\hline \multicolumn{4}{|l|}{$B M I, n(\%)$} \\
\hline Eutrophic & $13(46.4)$ & $14(53.6)$ & $0.064^{b}$ \\
\hline Overweight & $20(70.7)$ & $10(29.3)$ & \\
\hline Obese & $9(90)$ & $1(10)$ & \\
\hline \multicolumn{4}{|l|}{ Age, $n(\%)$} \\
\hline$<65$ years & $45(65.2)$ & $24(34.8)$ & $0.566^{b}$ \\
\hline$\geq 65$ years & $8(57.1)$ & $6(42.9)$ & \\
\hline \multicolumn{4}{|l|}{ Age range, $n(\%)$} \\
\hline$<40$ years & $6(66.7)$ & $3(33.3)$ & $0.406^{c}$ \\
\hline 40 to 60 years & $32(62.7)$ & $19(37.3)$ & \\
\hline 60 to 70 years & $10(66.7)$ & $5(33.3)$ & \\
\hline$>70$ years & $3(33.3)$ & $6(66.7)$ & \\
\hline \multicolumn{4}{|l|}{$\operatorname{Sex}, n(\%)$} \\
\hline Female & $32(62.7)$ & $19(37.3)$ & $0.790^{\mathrm{a}}$ \\
\hline Male & $21(65.6)$ & $11(34.4)$ & \\
\hline \multicolumn{4}{|l|}{ ASA classification, $n(\%)$} \\
\hline 1 & $24(60.0)$ & $16(40.0)$ & $0.481^{\mathrm{a}}$ \\
\hline 2 & $29(67.4)$ & $14(32.6)$ & \\
\hline \multicolumn{4}{|c|}{ Presence of comorbidity, n (\%) } \\
\hline Yes & $33(63.5)$ & $19(36.5)$ & $0.923^{b}$ \\
\hline No & $20(64.5)$ & $11(35.5)$ & \\
\hline \multicolumn{4}{|l|}{ Disease, $n$ (\%) } \\
\hline Malignant & $31(54.4)$ & $26(45.6)$ & $0.084^{b}$ \\
\hline Benign & $20(74.1)$ & $7(25.9)$ & \\
\hline \multicolumn{4}{|l|}{ Surgical time, $n$ (\%) } \\
\hline$\leq 3$ & $0(0.0)$ & $0(0.0)$ & \\
\hline$>3$ & $51(60.7)$ & $33(39.3)$ & - \\
\hline \multicolumn{4}{|l|}{ ICU time, n (\%) } \\
\hline Up to 1 day & $46(62.2)$ & $28(37.8)$ & $0.477^{c}$ \\
\hline More than 1 day & $7(77.8)$ & $2(22.2)$ & \\
\hline \multicolumn{4}{|l|}{ Use of opioids, $n$ (\%) } \\
\hline Yes & $41(62.1)$ & $25(37.9)$ & $0.517^{b}$ \\
\hline No & $12(70.6)$ & $5(29.4)$ & \\
\hline
\end{tabular}


Table 5 (continued)

Bold values indicate factors that negatively influenced the acceptance of SARP, in the univariate analysis

$S A R P$ simplified accelerated postoperative recovery program, $R C$ right colectomy, $T M E$ total mesorectal excision, $C D$ Clavien-Dindo, SD standard deviation, $Q 1 ; Q 3$ interquartile interval, ICU intensive care unit, ASA American Society of Anesthesiology

*Pearson's chi-square test

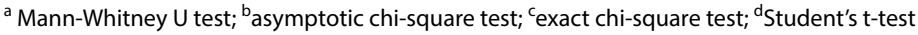

Table 6 Results of the multivariate analysis of the variables that influenced the tolerance or nontolerance of the SARP items $(n=84)$

\begin{tabular}{lcc}
\hline Variables & OR (95\% Cl OR) & P-value \\
\hline Type of surgery & $2.74(0.06 ; 121.57)$ & 0.602 \\
Complications (Clavien- & $14.78(3.42 ; 63.93)$ & $<\mathbf{0 . 0 0 0 1}$ \\
$\quad$ Dindo) & $1.01(1.002 ; 1.021)$ & $\mathbf{0 . 0 1 5}$ \\
Surgical time & $11.20(1.65 ; 75.89)$ & $\mathbf{0 . 0 0 1 3}$ \\
Need for ostomy & $1.04(0.98 ; 1.09)$ & 0.181 \\
Age & $1.84(0.51 ; 6.68)$ & 0.356 \\
Sex & $1.25(0.31 ; 4.99)$ & 0.757 \\
Neoplasm &
\end{tabular}

Bold values indicate factors that negatively influenced the acceptance of SARP, in the multivariate analysis

Hosmer-Lemeshow test $(p=0.930)$. Adjustment quality test of the logistic regression model

SARP simplified accelerated postoperative recovery program

participation of a senior surgeon in surgeries during the learning period, can not only contribute to reducing surgery time, but may also positively influence the implementation of SARPs.

In univariate analysis, another factor that significantly influenced the acceptance of the program was the type of surgery. Patients who underwent rectal surgeries had worse tolerance to the program than those who underwent colectomies. In patients who underwent colectomies, the acceptance rate of SARP was $78.6 \%$, while in patients who underwent a rectal resection, the acceptance rate decreased to $14.3 \%$. The ERAS Compliance Group [26] also reported poor results in patients who underwent rectal surgery, with a lower rate of adherence to the protocol, longer hospital stays, and higher readmission rates.

However, as demonstrated by multivariate analysis, the type of surgery itself was not statistically related to tolerance or non-tolerance to the implementation of the program. Nevertheless, patients who underwent rectal resections met the conditions associated with the worst acceptance of the SARP. In other words, they had longer surgeries, more postoperative complications and required ostomies more frequently, which were all variables that were significant in multivariate analysis.
Prospective randomized studies analyzing the impact of these and other variables in the acceptance of the accelerated recovery protocols will help to identify the conditions that lead to failure in up to $40 \%$ of patients [5-8]. As the multidisciplinary team addresses these specific issues, the cost-benefit analysis of such programs may improve.

Other variables that were analyzed, such as age, BMI, sex, and type of disease (whether benign or malignant), did not influence the acceptance of SARP. These data demonstrate that SARPs can be adopted even in elderly patients and in those with high BMI, as demonstrated previously by the study of the ERAS Compliance Group [26], which found no differences between these variables in the acceptance of the ERAS protocol.

The ERAS $^{\text {TM }}$ philosophy has gained wider acceptance in the scientific community [27]. Recently, the ERAS Society [16, 28], as well as the American Society of Colon and Rectal Surgeons and Society of American Gastrointestinal and Endoscopic Surgeons, published the guidelines for clinical practice for enhanced recovery after surgery in colorectal surgery [29]. In a meta-analysis of sixteen randomized, controlled studies, Greco et al. [30] demonstrated that hospital stays were shortened by 2.28 days and that the complication rate decreased by $40 \%$ with the adoption of accelerated postoperative recovery programs. Moreover, Lee et al. [4], among other authors, $[31,32]$, found that ERAS ${ }^{\mathrm{TM}}$ allowed patients to get back to work sooner and to be less dependent on caregivers, without impairing quality of life.

In Brazil, in a study with over 5,000 patients who underwent large abdominal surgeries, Bicudo-Salomão et al. [33] demonstrated that operation costs, length of hospital stay, and complication rates were reduced in patients submitted to the ACERTO ${ }^{\mathrm{TM}}$ accelerated recovery protocol, compared to patients submitted to conventional postoperative care.

However, for the programs to succeed, it is essential that measures be well-tolerated by the patients [34, 35]. In that regard, this study was able to identify variables that negatively influence patients' acceptance of SARPs. 


\section{Strengths}

It is randomized controlled trial. A simplified protocol of accelerated postoperative recovery was used, which can be easily implanted.

\section{Limitations}

The main limitations of the present study were a reduced number of patients for the comparative analysis of some variables, such as rehospitalization and reoperation rates, use of a single criterion (length of hospital stay) to define the success of the adopted program, and a lack of individual analysis of the acceptance or non-acceptance of each variable of the program.

\section{Conclusions}

The adoption of an accelerated recovery program, albeit simplified and focused on postoperative measures, was safe and brought benefits to patients tolerant to the program. In this study, the success rate in the implementation of the simplified accelerated recovery program was $60 \%$.

The development of complications, prolonged operative time, and the need for ostomy were variables that negatively impacted the success rate in the implementation of SARP. Randomized prospective studies are necessary to define the role of these and other possible variables in the non-tolerance by patients to accelerated recovery programs.

Therefore, the adoption of specific and targeted measures to reduce the negative impact of these variables on the acceptance of SARPs can lead to better acceptance by specific groups of patients, improving results and making them more cost-effective.

\begin{abstract}
Abbreviations
ACERTO $^{\mathrm{TM}}$ : Accelerated recovery protocol; ASA: American Society of Anesthesiologists; BMI: Body mass index; CC: Conventional postoperative care; DC: Dindo-Clavien; ERAS ${ }^{\mathrm{TM}}$ : Enhanced recovery after surgery; ICU: Intensive care unit; POD: Postoperative day; Q1; Q3: Interquartile range; SARP: Simplified accelerated recovery protocol; SD: Standard deviation; TME: Total mesorectal excision
\end{abstract}

\section{Acknowledgements}

Not applicable.

\section{Authors' contributions}

FLQ: data acquisition, analysis and interpretation; draft and critical revision of article for important intellectual content; final approval of version to be published. ALF: data analysis and interpretation; draft and critical revision of article for important intellectual content; critically revised article for important intellectual content, final approval of version to be published. ACA: data acquisition, analysis and interpretation. FHO: data acquisition, analysis and interpretation. PRFN: data acquisition. RAP: data acquisition. All authors read and approved the final manuscript.

\section{Funding}

Not applicable.

\section{Availability of data and materials}

The datasets generated and/or analysed during the current study are available in http://www.ensaiosclinicos.gov.br/rg/RBR-2b4fyrand from the corresponding author on reasonable request.

\section{Declarations}

\section{Ethics approval and consent to participate}

This study was approved by the Research Ethics Committee of our institution and by the Research Ethics Committee of the Federal University of Minas Gerais. It was registered on the Brazilian Platform of Research under CAAE number 43719015.4.0000.5149. It is enrolled in the Brazilian Clinical Trials Registry, linked to the International Clinical Trials Registry of the World Health Organization under number RBR-2b4fyr. All participants signed a statement of Informed Consent.

\section{Consent to publication}

Not applicable.

\section{Competing interests}

The authors declare that they have no competing interests.

\section{Author details}

${ }^{1}$ Colorectal Surgery Department, Hospital Felicio Rocho, Rua Felipe Dos Santos, 760, 501-3, Belo Horizonte, Minas Gerais CEP 30180160, Brazil. ²Department of Surgery at the School of Medicine, Federal University of Minas Gerais, Belo Horizonte, Brazil. ${ }^{3}$ School of Medicine, Federal University of Minas Gerais, Belo Horizonte, Brazil. ${ }^{4}$ Semper Hospital- Belo Horizonte, Belo Horizonte, Brazil. ${ }^{5}$ Department of Surgery, Federal University of São João del Rey, Divinópolis, Brazil. ${ }^{6}$ Hospital FelicioRocho, Belo Horizonte, Brazil.

Received: 21 July 2020 Accepted: 14 April 2021

Published online: 03 May 2021

\section{References}

1. Kehlet H. Multimodal approach to control postoperative pathophysiology and rehabilitation. Br J Anaesth. 1997;78:606-17.

2. Fearon $\mathrm{KC}$, et al. Enhanced recovery after surgery: a consensus review of clinical care for patients undergoing colonic resection. Clin Nutr. 2005;24:466-77.

3. Thanh NX, et al. An economic evaluation of the Enhanced Recovery After Surgery (ERAS) multisite implementation program for colorectal surgery in Alberta. Can J Surg. 2016;59:415-21.

4. Lee $L$, et al. Cost-effectiveness of enhanced recovery versus conventional perioperative management for colorectal surgery. Ann Surg. 2015;262:1026-33.

5. Stowers MD, Lemanu DP, Hill AG. Health economics in enhanced recovery after surgery programs. Can J Anaesth. 2015;62:219-30.

6. Lyon A, Payne CJ, Mackay GJ. Enhanced recovery program in colorectal surgery: does one size fit all? World J Gastroenterol. 2012;18:5661-3.

7. Springer JE, Doumouras AG, Lethbridge S, Forbes S, Eskicioglu C. A provincial assessment of the barriers and utilization of enhanced recovery after colorectal surgery. J Surg Res. 2019;235:521-8.

8. Vlug MS, et al. Laparoscopy in combination with fast track multimodal management is the best perioperative strategy in patients undergoing colonic surgery: a randomized clinical trial (LAFA-study). Ann Surg. 2011;254:868-75.

9. Moher D, et al. CONSORT 2010 explanation and elaboration: updated guidelines for reporting parallel group randomized trials. BMJ. 2010;340:c869.

10. Andersen J, et al. Readmission rates after a planned hospital stay of 2 versus 3 days in fast-track colonic surgery. Br J Surg. 2007;94:890.

11. Bardram L, Funch-Jensen $P$, Kehlet $H$. Rapid rehabilitation in elderly patients after laparoscopic colonic resection. Br J Surg. 2000;87:1540-5.

12. Gustafsson $\cup O$, et al. Adherence to the enhanced recovery after surgery protocol and outcomes after colorectal cancer surgery. ArchSurg. 2011;146:571-7. 
13. Dindo D, Demartines N, Clavien PA. Classification of surgical complications: a new proposal with evaluation in a cohort of 6336 patients and results of a survey. Ann Surg. 2004;240:205-13.

14. Nygren J, et al. An enhanced-recovery protocol improves outcome after colorectal resection already during the first year: a single-center experience in 168 consecutive patients. Dis Colon Rectum. 2009;52:978-85.

15. Teeuwen $\mathrm{PH}$, et al. Enhanced recovery after surgery versus conventional perioperative care in rectal surgery. Dis Colon Rectum. 2011;54:833-9.

16. Gustafsson UO, Scott MJ, Hubner M, et al. Guidelines for perioperative care in elective colorectal surgery: Enhanced Recovery After Surgery (ERAS ${ }^{\circledR}$ ) Society Recommendations: 2018. World J Surg. 2019;43:659-95.

17. Bona $S$, et al. Introducing an enhanced recovery after surgery program in colorectal surgery: a single center experience. World J Gastroenterol. 2014;20:17578-87.

18. Fonseca $L M$, et al. A simplified rehabilitation program for patients undergoing elective colonic surgery-randomized controlled clinical trial. Int J Colorectal Dis. 2011;26:609-16.

19. Aguilar-Nascimento JE, et al. Multimodal approach in colorectal surgery without mechanical bowel cleansing. Rev Col Bras Cir. 2009;36:204-9.

20. Cartmell MT, et al. A defunctioning stoma significantly prolongs the length of stay in laparoscopic colorectal resection. Surg Endosc. 2008;22:2643-7.

21. Ulrich $A B$, et al. Diverting stoma after low anterior resection: more arguments in favor. Dis Colon Rectum. 2009;52:412-8.

22. Delaney $C P$, et al. Prospective, randomized, controlled trial between a pathway of controlled rehabilitation with early ambulation and diet and traditional postoperative care after laparotomy and intestinal resection. Dis Colon Rectum. 2003;46:851-9.

23. Danielsen AK, Burcharth J, Rosenberg J. Patient education has a positive effect in patients with a stoma: a systematic review. Colorectal Dis. 2013;15:276-83.

24. Altuntas YE, et al. The role of group education on quality of life in patients with a stoma. Eur J Cancer Care (Engl). 2012;21:776-81.

25. Forsmo HM, Pfeffer F, Rasdal A, Sintonen H, Körner H, Erichsen C. Pre- and post operative stoma education and guidance within an enhanced recovery after surgery (ERAS) program reduces length of hospital stay in colorectal surgery. Int J Surg. 2016:3:121-6.

26. ERAS Compliance Group. The impact of enhanced recovery protocol compliance on elective colorectal cancer resection: results from an international registry. Ann Surg. 2015;261:1153-9.
27. Rauwerdink A, Jansen M, de Borgie CAJM, et al. Improving enhanced recovery after surgery (ERAS): ERAS APPtimize study protocol, a randomized controlled trial investigating the effect of a patient-centered mobile application on patient participation in colorectal surgery. BMC Surg. 2019;19(1):125.

28. Brindle M, Nelson G, Lobo DN, Ljungqvist O, Gustafsson UO. Recommendations from the ERAS Society for standards for the development of enhanced recovery after surgery guidelines. BJS Open. 2020;4(1):157-63.

29. Carmichael JC, Keller DS, Baldini G, Bordeianou L, Weiss E, Lee L, Boutros M, McClane J, et al. Clinical practice guidelines for enhanced recovery after colon and rectal surgery from the American Society of Colon and Rectal Surgeon and Society of American Gastrointestinal and Endoscopic Surgeons. Dis Colon Rectum. 2017;60:761-84.

30. Greco $M$, et al. Enhanced recovery program in colorectal surgery: a metaanalysis of randomized controlled trials. World J Surg. 2014;38:1531-41.

31. Smart NJ, et al. Deviation and failure of enhanced recovery after surgery following laparoscopic colorectal surgery: early prediction model. Colorectal Dis. 2012;14:727-34.

32. Steele SR, et al. Improving outcomes and cost-effectiveness of colorectal surgery. J Gastrointest Surg. 2014;18:1944-56.

33. Bicudo-Salomão A, et al. Impact of the ACERTO project in the postoperative morbi-mortality in a university hospital. Rev Col Bras Cir. 2011;38:3-10

34. Arrick L, Mayson K, Hong T, Warnock G. Enhanced recovery after surgery in colorectal surgery: impact of protocol adherence on patient outcomes. J Clin Anesth. 2018;21:7-12 (PMID:30583114)

35. van Rooijen S, Carli F, Dalton S, et al. Multimodal rehabilitation in colorectal cancer patients to improve functional capacity and reduce postoperative complications: the first international randomized controlled trial for multimodal prehabilitation. BMC Cancer. 2019;19(1):98.

\section{Publisher's Note}

Springer Nature remains neutral with regard to jurisdictional claims in published maps and institutional affiliations.
Ready to submit your research? Choose BMC and benefit from:

- fast, convenient online submission

- thorough peer review by experienced researchers in your field

- rapid publication on acceptance

- support for research data, including large and complex data types

- gold Open Access which fosters wider collaboration and increased citations

- maximum visibility for your research: over $100 \mathrm{M}$ website views per year

At BMC, research is always in progress.

Learn more biomedcentral.com/submissions 